\title{
3D QSAR of Pyrrolo Pyrimidine and Thieno Pyrimidines as Human Thymidylate Synthase Inhibitors
}

\author{
K. MEENA KUMARI , L.YAMINI, AND M.VIJJULATHA* \\ Molecular Modelling and Medicinal Chemistry Group, \\ Department of Chemistry, Nizam College, Osmania University, Hyderabad-500 001, India \\ vijjulathamanga@gmail.com
}

Received 08 September 2011; Accepted 11 November 2011

\begin{abstract}
Thymidylate synthase (TS) is a crucial enzyme for DNA biosynthesis and many nonclassical lipophilic antifolates targeting this enzyme are quite efficient and encouraging as antitumor drugs. We report 3D-QSAR analyses on pyrrolo pyrimidine and thieno pyrimidine antifolates to contemplate the mechanism of action and structure-activity relationship of these molecules. By applying leave-one-out (LOO) cross-validation study, cross-validated $\mathrm{q}^{2}$ value of 0.523 and 0.566 for CoMFA Ligand based(LB) and Receptor based(RB), 0.516 and 0.471 for CoMSIA LB and RB respectively. while the non-cross-validated $\mathrm{r}^{2}$ values were found to be 0.974 and 0.969 for CoMFA LB and RB, 0.983 and 0.972 for CoMSIA LB and RB respectively. The models were graphically interpreted using CoMFA and CoMSIA contour plots. The results obtained from this study were used for rational design of potent inhibitors against thymidylate synthase.
\end{abstract}

Key words: CoMFA, CoMSIA, pyrrolo pyrimidine, thieno pyrimidine, quantitative structure activity relationships, Thymidylate Synthase.

\section{Introduction}

Folate metabolism has long been recognised as an attractive target for cancer chemotherapy because of its indispensable role in the biosynthesis of nucleic acid precursors ${ }^{1,2}$. Thymidylate Synthase (TS) ${ }^{3}$ catalyses the reductive methylation of $2^{\prime}$-deoxyuridine $-5^{\prime}$ monophosphate (dUMP) to 2'-deoxythymidine-5'-monophosphate (dTMP) utilising 5, 10methylene tetrahydrofolate as the source of methyl group as well as the reductant ${ }^{4}$. This represents the sole denovo source of dTMP, hence inhibition of TS, in the development of antitumour agents.

Thymidylate Synthase (TS) is not a new target. However, there is active enthusiasm for the development of improved derivatives for TS specific inhibitors. Several TS inhibitors have been found in clinical utility as antitumour agents. Usually, 2-amino-4oxopyrimidine ring is considered important for potent TS inhibitory activity. Examples of 
clinically used TS inhibitors are raltitrexed (ZD1694) ${ }^{5}$, pemetrexed (alimta, LY231514) ${ }^{6}$ and $\mathrm{PDDF}^{2,7}$. The antifolate molecules evaluated in this investigation are derivatives of pyrrolo pyrimidine, thieno pyrimidines, having structures similar to the class of TS antifolates. Due to the interest in new anticancer drugs, several substituted pyrimidine inhibitors were chosen for screening against Human TS. A sound of understanding of the structural requirement for anticancer activity in substituted pyrimidines is important in guiding and optimising drug design efforts.

In the present study, the mechanism of intermolecular interaction between the most potent molecule 1 and TS was studied with the help of comparative molecular field analysis $(\text { CoMFA })^{8,9}$ and comparative molecular similarity indices analysis (CoMSIA) ${ }^{10}$ methodologies using the partial least squares (PLS) method ${ }^{11,12}$. CoMFA analysis involves the alignment of molecules in a structurally and pharmacologically reasonable manner on the basis of the assumption that each molecule acts via a common macromolecular target binding site. In this method, it is possible to predict the biological activity of molecules and represent the relationships between molecular properties (steric and electrostatic) and biological activity in the form of contour maps. CoMSIA approach calculates similarity indices in the space surrounding each of the aligned molecules in the dataset. CoMSIA is believed to be less affected by changes in molecular alignment and provides smooth and interpretable contour maps as a result of employing Gaussian type distance dependence with the molecular similarity indices it uses. Furthermore, in addition to steric and electrostatic fields of CoMFA, CoMSIA defines explicit hydrophobic and hydrogen bond donor and acceptor fields. Such 3D QSAR models would be of great help in a drug development program since the activity of new analogues could be quantitatively predicted before attempting their synthesis and testing.

\section{Experimental Section}

All pyrrolo pyrimidine and thieno pyrimidine derivatives and their invitro biological activity $\left(\mathrm{IC}_{50}\right)$ were taken from the literature ${ }^{13-18}$. The $\mathrm{IC}_{50}$ values in $\mu \mathrm{M}$ units were converted to the corresponding $\mathrm{pIC}_{50}$ values using the formula $\left(\mathrm{pIC}_{50}=-\operatorname{logIC} \mathrm{C}_{50}\right)($ Table 1$)$. The $\mathrm{pIC}_{50}$ values of the training set described in this manuscript span approximately $3.39 \log$ units. A total of 34 pyrrolo pyrimidine and thieno pyrimidine molecules were used as a data set, of which a set of 7 molecules were chosen as the test set, while the remaining 27 molecules were treated as a training set. The structures of all of the molecules are shown in (Fig. 1 and Table 1). The selected test set represented a range of inhibitory activity similar to that of a training set and was used to evaluate the predictive power of the CoMFA and CoMSIA models.

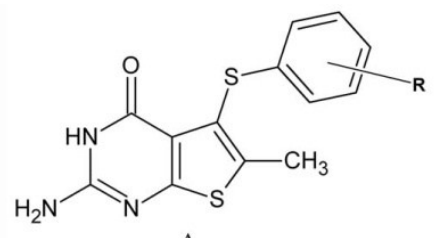

A

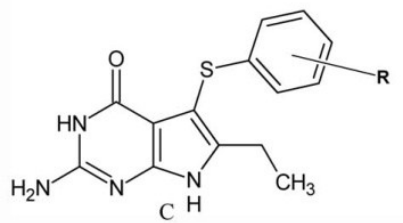

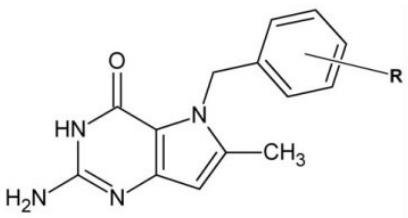

B

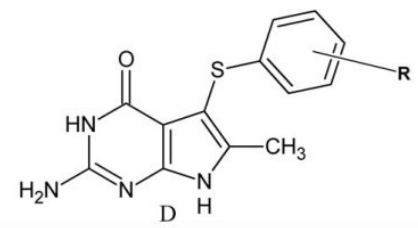

Figure 1. Core structures of pyrrolo pyrimidine and thieno pyrimidine molecules. 
Table 1. Structures of the training and test set of molecules showing Experimental and Predicted activities.

\begin{tabular}{|c|c|c|c|c|c|c|c|c|}
\hline \multirow{2}{*}{ Molecule } & \multirow{2}{*}{ Core } & \multirow{2}{*}{$\mathrm{R}$} & \multirow{2}{*}{$\begin{array}{l}\mathrm{IC}_{50} \\
(\mu \mathrm{M})\end{array}$} & \multirow{2}{*}{$\mathrm{pIC}_{50}$} & \multicolumn{2}{|c|}{ LB Activity } & \multicolumn{2}{|c|}{ RB Activity } \\
\hline & & & & & CoMFA & CoMSIA & CoMFA & CoMSIA \\
\hline 1. & A & & 0.04 & 7.400 & 7.385 & 7.384 & 7.322 & 7.406 \\
\hline 2. & A & $\mathrm{H}$ & 1.2 & 5.920 & 5.868 & 5.990 & 6.081 & 5.946 \\
\hline 3. & A & $4-\mathrm{Cl}$ & 0.26 & 6.585 & 6.614 & 6.583 & 6.661 & 6.540 \\
\hline 4. & A & $4-\mathrm{NO}_{2}$ & 0.11 & 6.958 & 6.997 & 7.001 & 6.972 & 7.068 \\
\hline 5. & A & 2,5-OMe & 4.6 & 5.337 & 5.340 & 5.384 & 5.422 & 5.374 \\
\hline 6. & A & $3,4-\mathrm{Cl}$ & 0.11 & 6.958 & 6.979 & 6.851 & 6.952 & 6.807 \\
\hline$* 7$ & A & $\begin{array}{r}3,4(-\mathrm{CH}=\mathrm{CH}- \\
\mathrm{CH}=\mathrm{CH}-)\end{array}$ & 0.12 & 6.920 & 5.859 & 6.580 & 6.288 & 6.719 \\
\hline 8. & A & $\mathrm{F}$ & 1.1 & 5.958 & 5.802 & 5.921 & 5.748 & 5.909 \\
\hline 9. & $\mathrm{~B}$ & & 0.046 & 7.337 & 7.324 & 7.323 & 7.395 & 7.301 \\
\hline 10. & B & $\mathrm{H}$ & 20 & 4.698 & 4.805 & 4.597 & 4.665 & 4.579 \\
\hline 11. & & & 2.3 & 5.638 & 5.727 & 5.471 & 5.679 & 5.362 \\
\hline 12. & $\mathrm{~B}$ & $4-\mathrm{F}$ & 3.7 & 5.431 & 5.794 & 5.791 & 5.560 & 5.847 \\
\hline 13. & $\mathrm{~B}$ & $4-\mathrm{Cl}$ & 2.9 & 5.537 & 5.598 & 5.349 & 5.614 & 5.396 \\
\hline 14. & B & $4-\mathrm{NO}_{2}$ & 1.4 & 5.853 & 5.847 & 5.607 & 5.637 & 5.674 \\
\hline 15. & B & $4-\mathrm{OMe}$ & 1.5 & 5.832 & 5.553 & 5.838 & 5.727 & 5.913 \\
\hline 16. & B & $4-\mathrm{OCF}_{3}$ & 1.3 & 5.886 & 5.997 & 5.928 & 5.937 & 5.751 \\
\hline 17. & B & $3,5-\mathrm{Br}$ & 10 & 5.000 & 4.790 & 5.205 & 4.912 & 5.209 \\
\hline 18. & B & $3,4-\mathrm{Cl}$ & 5.4 & 5.267 & 5.222 & 5.455 & 5.390 & 5.512 \\
\hline 19. & $\mathrm{C}$ & & 0.09 & 7.045 & 7.041 & 7.062 & 6.999 & 7.092 \\
\hline 20. & $\mathrm{C}$ & $3,4-\mathrm{Cl}$ & 0.23 & 6.638 & 6.550 & 6.648 & 6.720 & 6.589 \\
\hline 21. & $\mathrm{C}$ & $4-\mathrm{NO}_{2}$ & 0.24 & 6.619 & 6.496 & 6.734 & 6.555 & 6.758 \\
\hline 22. & $\mathrm{C}$ & $4-F$ & 2.7 & 5.568 & 5.730 & 5.555 & 5.658 & 5.506 \\
\hline$*_{23}$ & $\mathrm{C}$ & $4-\mathrm{Br}$ & 1.0 & 6.000 & 5.791 & 5.484 & 6.043 & 5.388 \\
\hline$* 24$ & $\mathrm{C}$ & $3-\mathrm{Br}$ & 1.1 & 5.958 & 6.285 & 6.255 & 6.314 & 6.180 \\
\hline$* 25$ & $\mathrm{C}$ & 2,6-Me & 26 & 4.585 & 5.363 & 4.616 & 5.421 & 4.770 \\
\hline$* 26$ & $\mathrm{C}$ & $\begin{array}{c}2,3(-\mathrm{CH}=\mathrm{CH}- \\
\mathrm{CH}=\mathrm{CH}-)\end{array}$ & 5.6 & 5.251 & 5.028 & 5.016 & 5.339 & 5.026 \\
\hline
\end{tabular}




\begin{tabular}{|c|c|c|c|c|c|c|c|}
\hline *27. & $\begin{array}{c}3,4(-\mathrm{CH}=\mathrm{CH}- \\
\mathrm{CH}=\mathrm{CH}-)\end{array}$ & 5.6 & 5.251 & 5.637 & 5.447 & 6.162 & 5.627 \\
\hline 28. & & 0.34 & 6.468 & 6.446 & 6.419 & 6.663 & 6.390 \\
\hline 29. & $\mathrm{D}$ & 30 & 4.522 & 4.784 & 4.594 & 4.925 & 4.724 \\
\hline 30. & $4-\mathrm{Cl}$ & 1.0 & 6.000 & 5.641 & 5.811 & 5.529 & 5.806 \\
\hline *31. & $\begin{array}{r}3,4(-\mathrm{CH}=\mathrm{CH}- \\
\mathrm{CH}=\mathrm{CH}-)\end{array}$ & 2.0 & 5.698 & 5.556 & 5.746 & 5.456 & 6.037 \\
\hline 32. & $\mathrm{D}$ & 0.54 & 7.267 & 7.361 & 7.271 & 7.210 & 7.182 \\
\hline 33. & D & 0.051 & 7.292 & 7.310 & 7.300 & 7.341 & 7.373 \\
\hline 34. & & 100 & 4.000 & 4.015 & 4.014 & 3.923 & 4.000 \\
\hline
\end{tabular}

*represent test set of molecules.

All molecular modeling calculations were performed on a Linux operating system. Three dimensional structure building and all modeling were performed using the SYBYL -X 1.2 molecular modeling program package ${ }^{19}$. Gasteiger-Hückel charges were assigned and then energy minimization of each molecule was performed using the conjugate gradient method and Tripos FF standard force field with a distance-dependent dielectric function. The minimization was terminated when the energy gradient convergence criterion of 0.001 $\mathrm{kcal} \mathrm{mol}{ }^{-1} \cdot \AA^{-1}$ was reached. For ligand based 3D QSAR, the low energy conformation thus obtained was then aligned on most active molecule in the series, molecule 1 using ALIGN DATABASE command in SYBYL -X 1.2 taking the substructure that is common to all. The resulting alignment model (Figure $2 \mathrm{~b}$ ) was then subjected to CoMFA and CoMSIA studies. For receptor based alignment best docked mode of the most active molecule 1 was taken as template. All minimized structures were aligned on this template to get the molecular alignment for receptor based CoMFA and CoMSIA (Figure 2c). The accuracy of the prediction of CoMFA and CoMSIA models and the reliability of the contour models depend strongly on the structural alignment of the molecules. 
<smiles>Nc1nccc(=O)[nH]1</smiles>

(a)

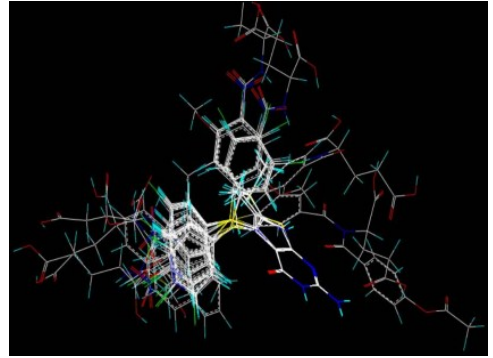

(b)

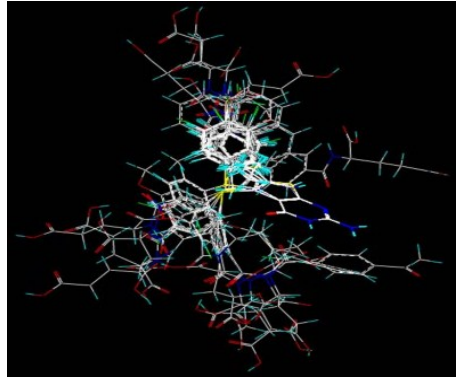

(c)

Figure 2. (a) Common substructure used for alignment. (b) Ligand-based alignment on common substructure (c) Receptor-based alignment on common substructure.

The X-ray structure of TS was obtained from protein data bank (1JU6) which was used as receptor site. All water molecules were removed and the protein was minimized using Tripos force field by applying Gasteiger Huckel charges using conjugate gradient method until the energy gradient convergence criterion with $0.05 \mathrm{kcal} / \mathrm{mol} / \AA$. The active site was defined having amino acids Arg50, Glu87, Trp109, Asn112, Tyr135, Cys195, His196, Gln214, Arg215, Ser216, Asp218, Phe225, Asn226, His256, Tyr258 ${ }^{20}$. The most active molecule 1 was docked into the monomer unit (A) of TS and the dock pose was used as the template onto which the dataset was aligned.

Standard Tripos force field was employed for the Comparative Molecular Field Analysis (CoMFA) and Comparative Molecular Similarity Indices (CoMSIA) analysis. A 3D cubic lattice overlapping all entered molecules and extended by at least $4 \AA$ in each direction with each lattice intersection of a regularly spaced grid of $2.0 \AA$ was created.

The steric and electrostatic parameters were calculated in case of the CoMFA fields, while $\mathrm{H}$-bond donor parameters in addition to steric and electrostatic were calculated in case of the CoMSIA fields at each lattice. $\mathrm{A} \mathrm{sp}^{3}$ hybridized carbon atom was used as a probe atom to generate steric (Lennard-Jones potential) field energies and a charge of +1 to generate electrostatic (coulombic potential) field energies. A distance dependent dielectric constant of 1.00 was used. The steric and electrostatic fields were truncated at $+30.00 \mathrm{kcal} / \mathrm{mol}$. The similarity indices descriptors were calculated using the same lattice box employed for CoMFA calculations, using $\mathrm{sp}^{3}$ carbon as a probe atom with $\mathrm{a}+1$ charge, +1 Hbond donor and attenuation factor of 0.3 for the Gaussian type distance.

A partial least squares regression was used to generate a linear relationship that correlates changes in the computed fields with changes in the corresponding experimental values of biological activity $\left(\mathrm{pIC}_{50}\right)$ for the data set of ligands. Biological activity values of ligands were used as dependent variables in a PLS statistical analysis. The column filtering value (s) was set to $2.0 \mathrm{kcal} / \mathrm{mol}$ to improve the signal-to-noise ratio by omitting those lattice points whose energy variations were below this threshold. Cross-validations were performed by the leave-one-out $(\mathrm{LOO})^{9,21}$ procedure to determine the optimum number of components (ONC) and the coefficient $\mathrm{q}^{2}$. The optimum number of components obtained is then used to derive the final QSAR model using all of the training set molecules with non-cross validation and to obtain the conventional correlation coefficient $\left(\mathrm{r}^{2}\right)$. To validate the CoMFA and CoMSIA derived models, the predictive ability for the test set of molecules (expressed as $r^{2}$ pred) was determined by using the following equation:

$$
r_{\text {pred }}^{2}=(\mathrm{SD}-\mathrm{PRESS}) / \mathrm{SD}
$$

$\mathrm{SD}$ is the sum of the squared deviations between the biological activities of the test set molecules and the mean activity of the training set molecules. PRESS is the sum of the squared deviation between the observed and the predicted activities of the test set molecules. 
Since the statistical parameters were found to be the best for the model from the LOO method, it was employed for further predictions of the designed molecules.

For stronger evaluation of the model applicability on new chemicals, the activities of the new molecules were evaluated using these QSAR models.

\section{Results and Discussions}

The 3D QSAR - CoMFA and CoMSIA analyses were carried out using pyrrolo pyrimidine and thienopyrimidine derivatives reported as potent Human Thymidylate Synthase inhibitors by Aleem Gangjee et. al. Molecules with precise $\mathrm{IC}_{50}$ values were selected, a set of 34 molecules were used for derivation of model, these were divided into training set of 27 molecules and test set of 7 molecules.

The CoMFA and CoMSIA statistical analyses are summarised in Table 2. Statistical data shows $\mathrm{q}_{\text {loo }}^{2} 0.523$ and 0.566 for CoMFA ligand based(LB) and receptor based(RB), 0.516 and 0.471 for CoMSIA ligand based(LB) and receptor based(RB) respectively. The $\mathrm{r}^{2}{ }_{\text {ncv }}$ of 0.974 and 0.969 for CoMFA LB and RB, 0.983 and 0.969 for CoMSIA LB and RB respectively, that includes a good internal predictive ability of the models. To test the predictive ability of the models a test set of seven molecules excluded from the model generation were used. The predictive correlation coefficient $r_{\text {pred }}^{2}$ of 0.505 and 0.50 for CoMFA LB and RB, 0.866 and 0.810 for CoMSIA LB and RB respectively indicates good external predictive ability of the models. The graph for the actual and predictive $\mathrm{pIC}_{50}$ for training and test set of CoMFA LB and RB and CoMSIA LB and RB studies shown in Fig. 3(a,b,c,d). The CoMSIA models showed better results than CoMFA models, this shows that the Hbond donor fields that are not included in the CoMFA model are important for explaining the potency of the molecules. The observed and predictive activity of the molecules is provided in Table 1 .

Table 2. Summary of CoMFA and CoMSIA results.

\begin{tabular}{|c|c|c|c|c|}
\hline \multirow[t]{2}{*}{ PLS Statistics } & \multicolumn{2}{|c|}{ Ligand Based } & \multicolumn{2}{|c|}{ Receptor Based } \\
\hline & CoMFA & CoMSIA & CoMFA & CoMSIA \\
\hline $\mathrm{r}_{\mathrm{ncv}}^{2} \mathrm{a}$ & 0.974 & 0.983 & 0.969 & 0.972 \\
\hline $\mathrm{SEE}^{\mathrm{b}}$ & 0.178 & 0.153 & 0.195 & 0.196 \\
\hline $\mathrm{F}_{\text {test }}{ }^{\mathrm{c}}$ & 96.591 & 92.216 & 70.581 & 55.831 \\
\hline $\mathrm{r}_{\mathrm{cv}}^{2 \mathrm{~d}}$ & 0.523 & 0.516 & 0.566 & 0.471 \\
\hline$r_{\text {pred }}^{2}$ & 0.5052 & 0.8665 & 0.5003 & 0.8106 \\
\hline PLS Components ${ }^{\mathrm{f}}$ & 7 & 10 & 8 & 10 \\
\hline \multicolumn{5}{|c|}{ Fraction of field contributions } \\
\hline Steric & 0.516 & 0.296 & 0.488 & 0.282 \\
\hline Electrostatic & 0.484 & 0.557 & 0.512 & 0.578 \\
\hline H-Bond Donor & --- & 0.147 & --- & 0.140 \\
\hline
\end{tabular}

${ }^{\mathrm{a}}$ Correlation coefficient ${ }^{\mathrm{b}}$ Standard error of estimate ${ }^{\mathrm{c}}$ Fisher test value ${ }^{\mathrm{d}}$ Cross validated correlation coeffient by leave-one-out method ${ }^{\mathrm{e}}$ Predicted correlation coefficients on test set

${ }^{\mathrm{f}}$ Optimum number of principal components. 


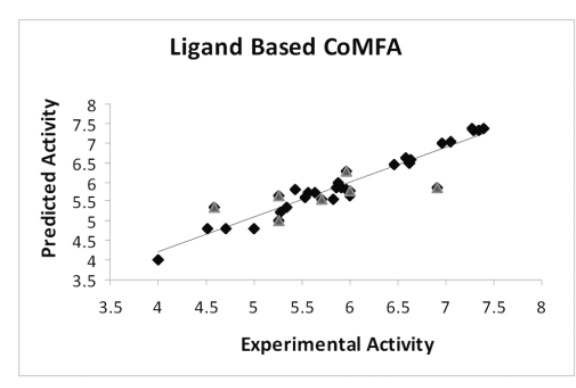

(a)

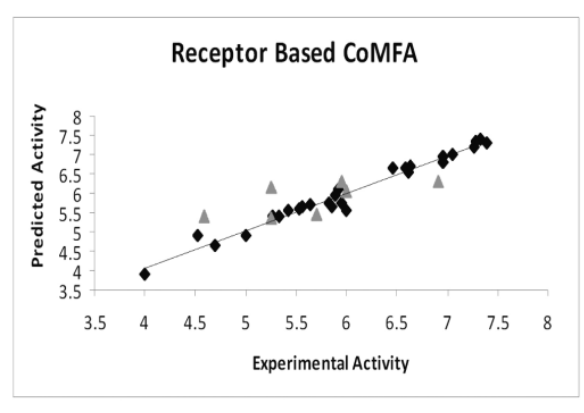

(b)

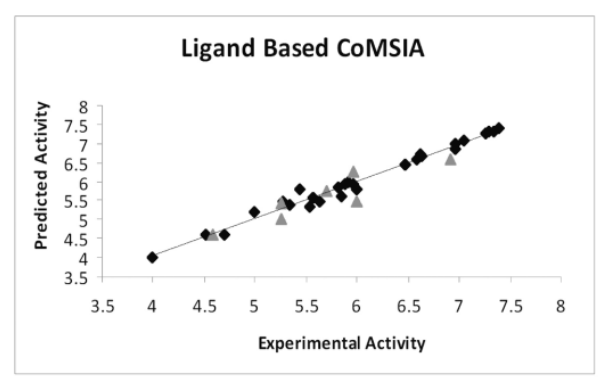

(b)

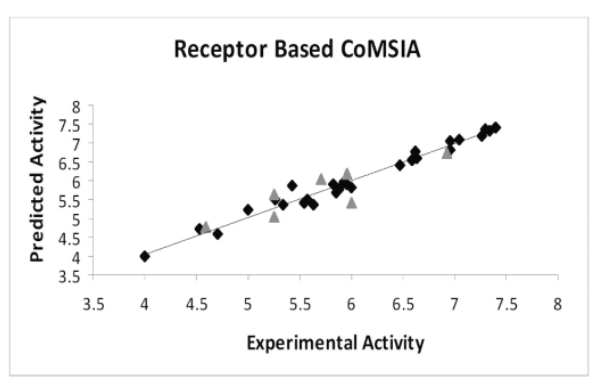

(d)

Figure 3. Scatter plot of observed versus predicted $\mathrm{pIC}_{50}$ values for the training set ( $\left.\mathbf{\square}\right)$ and the test set $(\Delta)$ molecules based on (a)Ligand based CoMFA model,(b) Ligand based CoMSIA model, (c) Receptor based CoMFA model and (d) Receptor based CoMSIA model.

To visualise the information content of the derived 3D QSAR models, CoMFA and CoMSIA contour maps were generated. The contour plots are the representation of the lattice points and the difference in the molecular field values at lattice point strongly connected with the difference in the receptor binding affinity. Molecular fields define the favourable and unfavourable interaction energies of aligned molecules with a probe atom traversing across the lattice points suggesting the modifications required to design new molecules. The contour maps of CoMFA denote the region in the space where the molecules would be favourably or unfavourably interact with the receptor, while the CoMSIA contour maps denote areas within the specified region where the presence of a group with a particular physicochemical property binds to the receptor. The CoMFA/CoMSIA results were graphically interpreted by field contribution maps using the 'STDEV COEFF' field type.

Figure 4(a,b) and 5(a,b) shows the contour maps for LB and RB CoMFA derived from the CoMFA PLS models. 


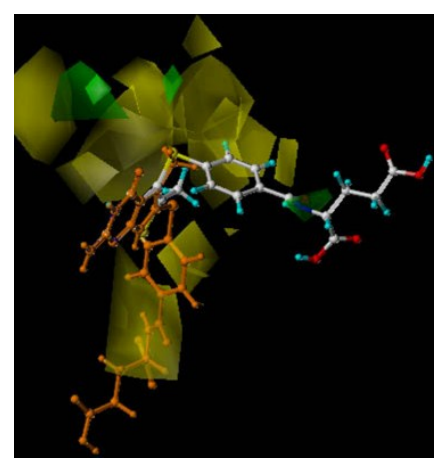

Figure 4(a)

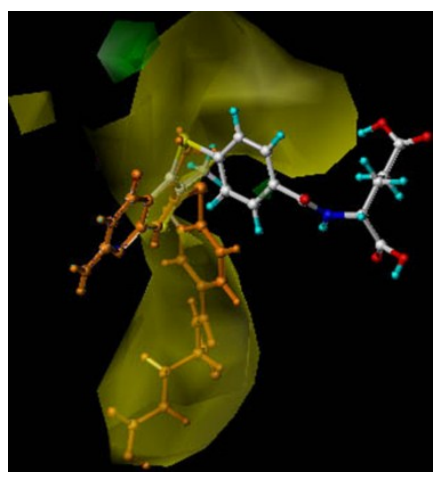

Figure 5(a)

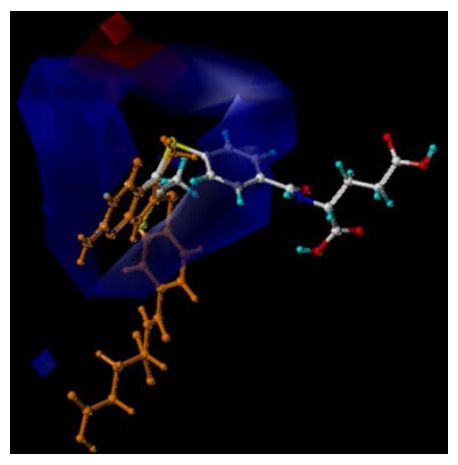

Figure 4 (b)

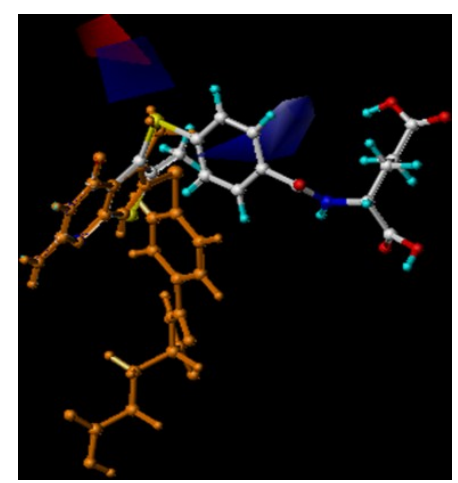

Figure 5(b)

Figure 4(a,b) and 5(a,b). CoMFA contour maps for LB and RB displayed with most potent molecule 1 (coloured by atom type) and less potent molecule 34 (orange coloured). (a) Steric contour maps for LB and RB CoMFA. (b) Electrostatic contour maps for LB and RB CoMFA.

The most potent molecule 1 (coloured atom type) and the least active molecule 34(coloured orange) were embedded in the map to demonstrate their affinities for the steric and electrostatic regions of inhibitors. The area of yellow indicates region of steric hindrance to activity, while green areas indicate a steric contribution to potency. The blue region indicates positive electrostatic charge potential associated with increased activity, while red region shows negative charge potential.

All the contours represent the default 80 and $20 \%$ level contribution for favoured and disfavoured regions respectively. The CoMFA steric and electrostatic LB and RB, where the highest activity of molecule 1 and the least activity of molecule 34 shown in Fig. 4(a,b) and $5(a, b)$ can be attributed by the presence of large yellow contour around the glutamic acid side chain in the molecule 34 , and in molecule 1 the glutamic acid side chain is away from the yellow disfavoured region due to the change in the position of the glutamic acid side chain from $\mathrm{S} 11$ to $\mathrm{S} 12$ as shown in Fig.6. The $-\mathrm{C}=0$ group attached to the benzene ring is oriented towards red region indicating favoured negative electrostatic potentials. The presence of green contour on the $-\mathrm{NH}$ region of glutamic acid suggest substitution with 
bulkier group should increase the activity. All these explain the high activity of the molecule 1 .
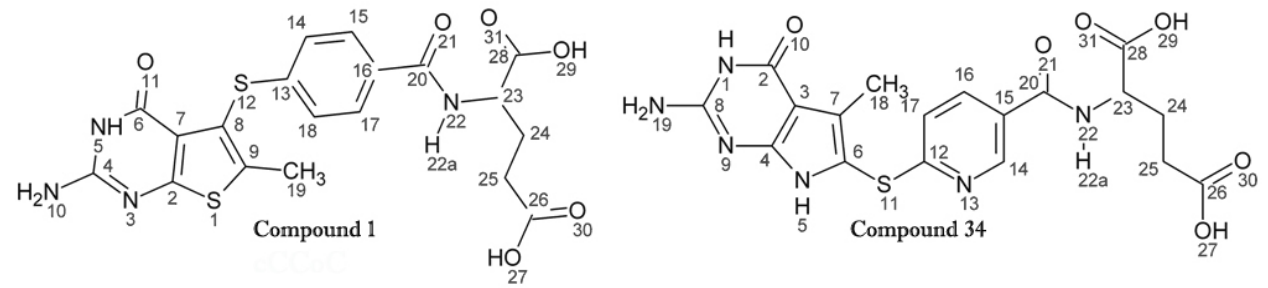

Figure 6. Showing change in the position of S12 to S11 from molecule 1 to molecule 34.

The CoMSIA steric and electrostatic contour maps shown in Fig.7(a,b) and 8(a,b) are similar to the CoMFA contour maps hence not discussed. The Hbond donor contour map of the CoMSIA model along with most and least potent molecule 1 and 34 is depicted in Fig. $7(\mathrm{c})$ and $8(\mathrm{c})$. The least potent molecule 34 is protruding into the hydrogen bond donor disfavoured contour while the potent molecule 1 due to the change in the position of the glutamic acid side chain from S11 to S12 has shifted away from the disfavoured region. Hence it can be analysed that positioning of glutamic acid side chain has a important role in increasing the activity of the molecule.

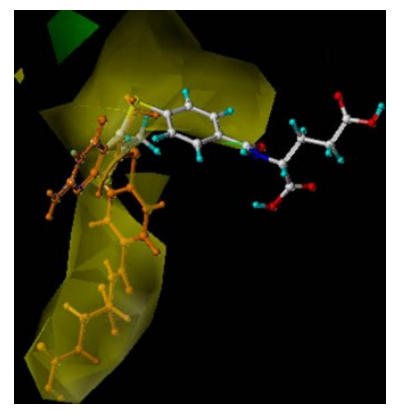

Figure 7(a)

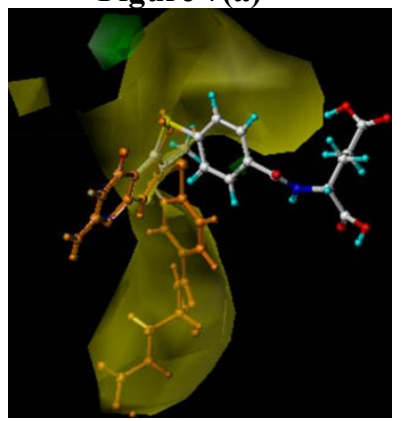

Figure 8 (a)

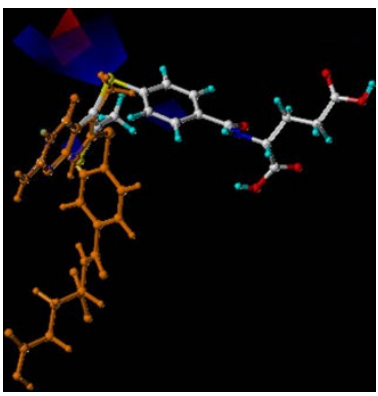

Figure 7(b)

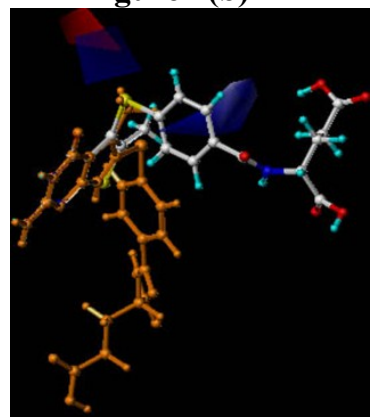

Figure 8(b)

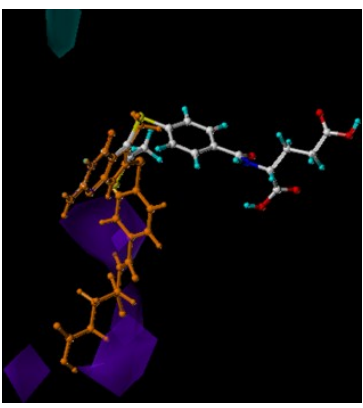

Figure 7(c)

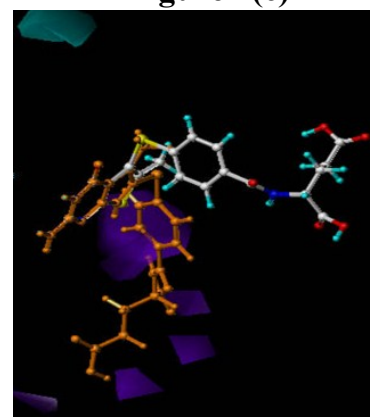

Figure 8 (c)

Figure $7(\mathbf{a}, \mathbf{b}, \mathbf{c})$ and $8(\mathbf{a}, \mathbf{b}, \mathbf{c})$. CoMSIA contour maps for LB and RB displayed with most potent molecule 1 (coloured by atom type) and less potent molecule 34 (orange coloured). (a) Steric contour maps for LB and RB CoMSIA. (b) Electrostatic contour maps for LB and RB CoMSIA. (c) Hydrogen bond donor contour maps for LB and RB CoMSIA. 


\section{Design of New Inhibitors}

The detailed contour map analysis of both CoMFA and CoMSIA models empowered us to identify the structural requirement for the observed inhibitory activity. Steric favoured and electro negative groups have been substituted on the $\mathrm{NH}$ of the glutamic acid side chain with 6 different substituents that gave a good predictive inhibitory activity values for the CoMSIA model and comparable predictive inhibitory activity values for the CoMFA model. Docking studies showed that the existing high active molecule had four hydrogen bonds (3HB- ASN226, 1HB-ASP218) while the designed molecules showed ten hydrogen bonds(2HB-ARG50, 1HB-GLU87, 1HB-ASN226, 5HB-ARG215, 1HB-SER216) as shown in figure 9. This analysis also predicts that the designed molecules have better interactions than the high active molecule. The structures and their predicted activity values along with calculated $\mathrm{IC}_{50}$ values for both CoMFA and CoMSIA models have been given in Fig. 10 and Table 3 .

Table 3. The LB and RB Predicted activity values of newly designed molecules.

$$
\text { LB Predicted Activity RB Predicted Activity }
$$

Molecule

$$
\mathrm{R}
$$

$$
\begin{array}{llll}
\text { CoMFA CoMSIA CoMFA CoMSIA } & \begin{array}{l}
\text { Calculated } \\
\mathrm{IC}_{50}(\mu \mathrm{M})
\end{array}
\end{array}
$$

New1<smiles>CC(=O)C(C)Br</smiles>

$$
8.139
$$

7.080

8.031

0.0092

New2<smiles>CN1C(=O)CCC1=O</smiles>

7.040

8.126

7.058

7.935

0.0074

New3<smiles>Cc1c[nH]c(=O)[nH]c1=O</smiles>

7.338

7.981

7.182

7.906

0.0104

New4<smiles>CC=C(C)C</smiles>

7.366

\subsection{1}

7.151

7.873

0.0070

New5

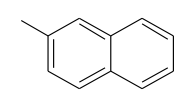

7.163

7.862

7.150

7.718

0.0136

New6

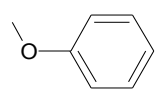

7.371

7.692

7.007

7.586

0.0202 


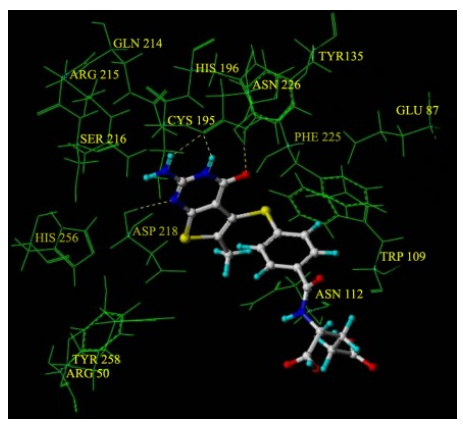

(a)

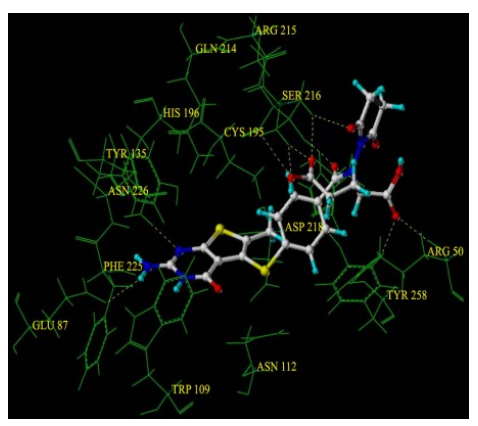

(b)

Figure 9: (a) Dockpose of molecule 1 (b) Dockpose of designed molecule (New 2).<smiles>[R]N(C(=O)c1ccc(Sc2c(C)sc3nc(N)[nH]c(=O)c23)cc1)C(CCC(=O)O)C(=O)O</smiles>

Figure 10: Structure of newly designed molecule.

\section{Conclusions}

Our present studies have established that CoMFA and CoMSIA models are quite reliable to efficiently guide further modification in the molecules for obtaining better drugs. They have provided good statistical results in terms of $\mathrm{q}^{2}$ and $\mathrm{r}^{2}$ values for pyrrolo pyrimidine and thieno pyrimidine antifolate derivatives. Both CoMFA and CoMSIA models provided the significant correlations of biological activities with steric, electrostatic and Hbond donor fields, establishing the significance of these fields in the selectivity and activity of the molecules. The 3D-QSAR results revealed some important sites, where steric, hydrogenbond donor modifications should significantly affect the bioactivities of the molecules. Using these clues novel human thymidylate synthase inhibitors with high affinity were designed that can be potent molecules for the treatment of cancer.

\section{Acknowledgements}

We gratefully acknowledge support for this research from Council of Scientific and Industrial Research (Project No. 01/(2436)/10/EMR-II) and providing SRF for the research scholar, University Grants Commission India, Department of Science and Technology, India and Department of chemistry, Nizam College, Hyderabad. 


\section{References}

1. Kisliuk R L, The Biochemistry of Folates, Academic Press; New York, 1984.

2. Berman E M and Werbel L M, J. Med. Chem. 1991, 34, 479.

3. Carreras C W and Santi S V, Annu. Rev. Biochem. 1995, 64, 721.

4. Pogolotti A L and Santi D V, Chem. 1977, 1, 277.

5. Jackman A L, Taylor G A, Gibson W, Kimbell R, Brown M, Calvert A H, Judson I R and Hughes L R, Cancer Res. 1991, 51, 5579.

6. Taylor E C, Kuhnt D, Shih C, Rinzel S M, Grindey G B, Barredo J, Jannatipour M and Moran R A, J. Med. Chem. 1992, 35, 4450.

7. Douglas K T, Med. Res. Rev. 1987, 7, 441.

8. Cramer R.DIII, Patterson D E and Bunce J D, J. Am. Chem. Soc. 1988, 110,5959.

9. Cramer R DIII, Bunce J D, Patterson D E, Quant. Struct. Act. Relat. 1988, 7, 18.

10. Klebe G, Abraham U and Mietzner T, J. Med. Chem. 1994, 37, 4130.

11. Wold S, Albano C, Dunn W J, Edlund U, Esbenson K and Geladi P, Chemometrics, Kowalski B (ed) Reidel, Dordrecht, The Netherlands, 1984

12. Staahle L and Wold S, J. Chemom. 1987, 1, 185.

13. Aleem Gangjee, Yibin Qiu, Wei Li and Roy Kisliuk L, J. Med. Chem. 2008, 51, 5789.

14. Aleem Gangjee, Wei Li, Jie Yang and Roy Kisliuk L, J. Med. Chem. 2008, 5,168.

15. Aleem Gangjee, Hitesh Kumar D, Jain, Jaclyn phan, Xin Lin, XiÅhong, John J, McGuire, Roy Kisluik L, J. Med. Chem. 2006, 49, 1055.

16. Aleem Gangjee, Farahnaz Mavandadi, Roy Kisluik L, John J, McGuire, Sherry and Queener F, J. Med. Chem. 1996, 39, 4563.

17. Aleem Gangjee, HiteshKumar D, Jain J, John McGuire and Roy Kisluik L, J. Med. Chem. 2004, 47, 6730.

18. Aleem Gangjee, Xin Lin, Roy Kisluik L, John McGuire J, J. Med. Chem. 2005, 48, 7215.

19. Sybyl-X1.2, Tripos Associates, St. Louis (MO), 2009.

20. Kumari K M, Kanth S S, Vijjulatha M, Internet Electron. J. Mol. Des. 2008, 7, 131.

21. Wold S, Technometrics, 1978, 4, 397. 


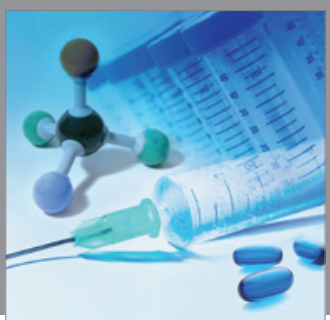

International Journal of

Medicinal Chemistry

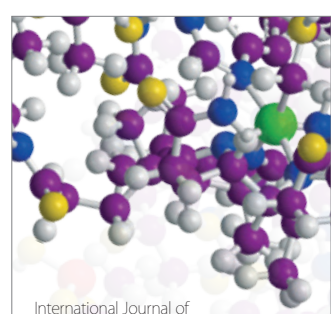

Carbohydrate Chemistry

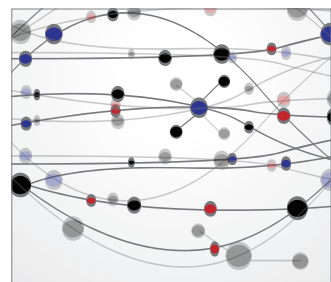

The Scientific World Journal
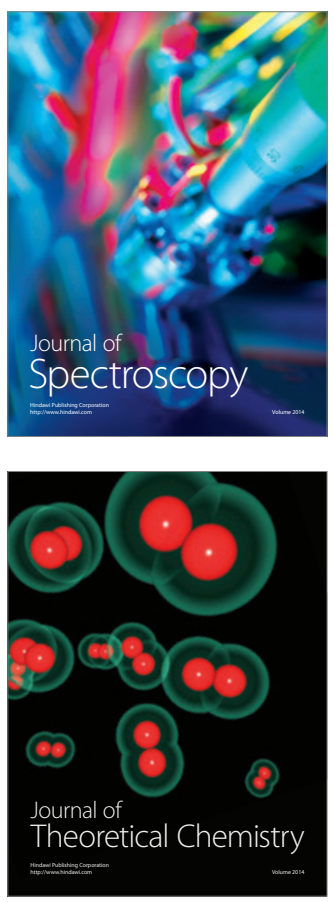
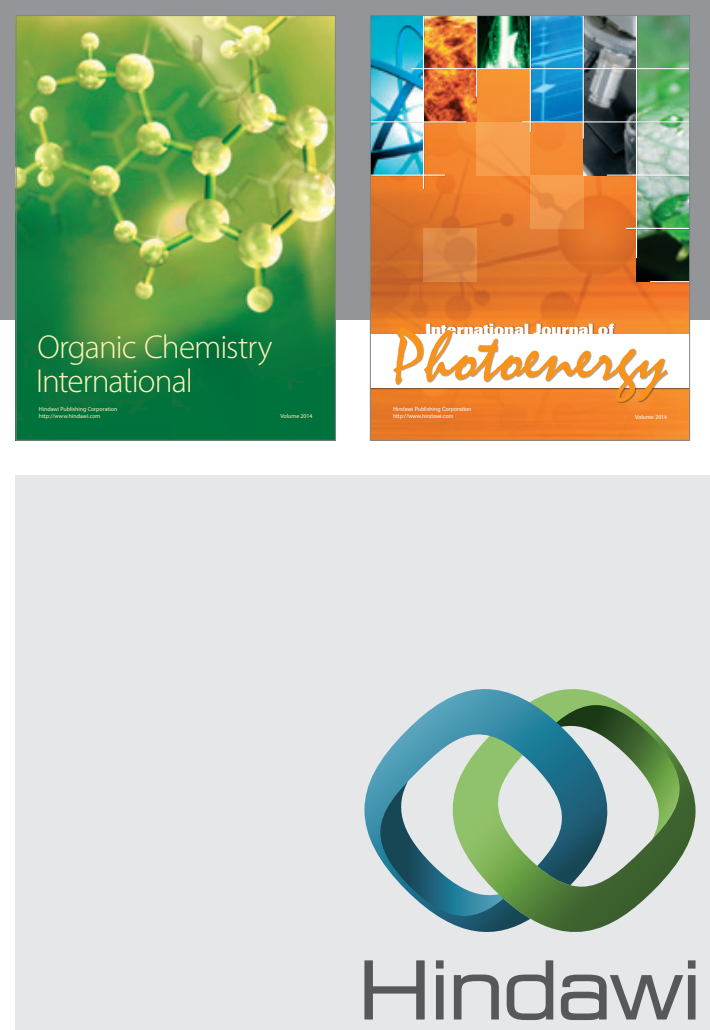

Submit your manuscripts at

http://www.hindawi.com
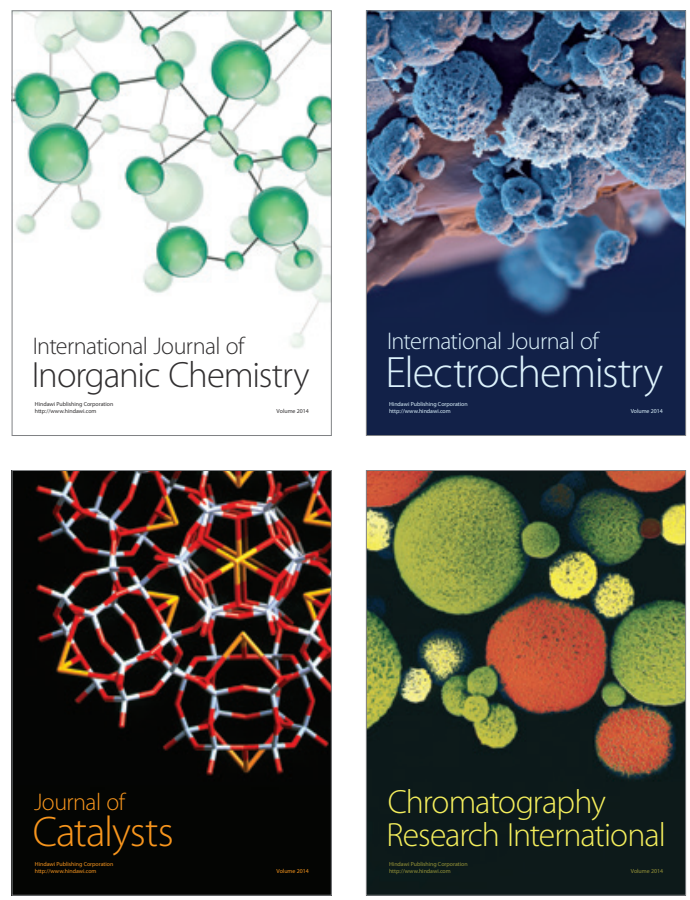
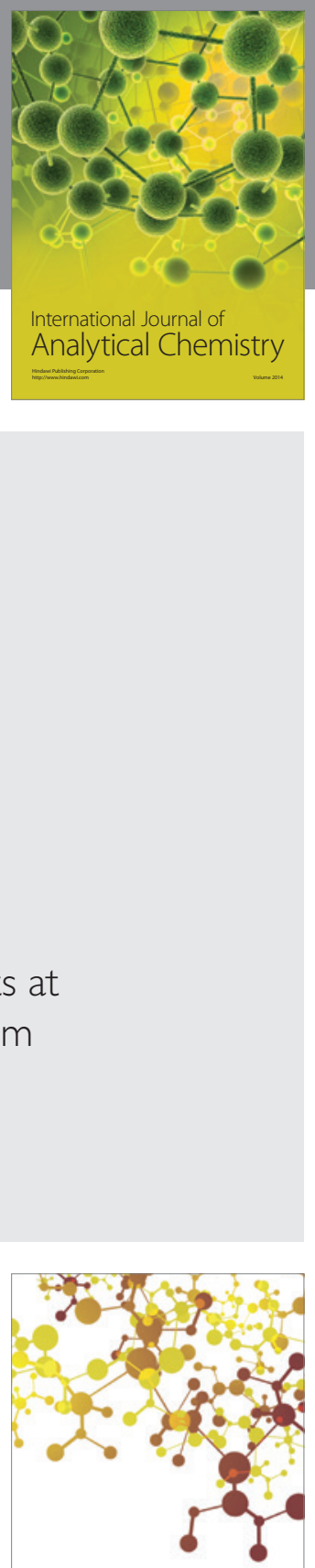

Journal of

Applied Chemistry
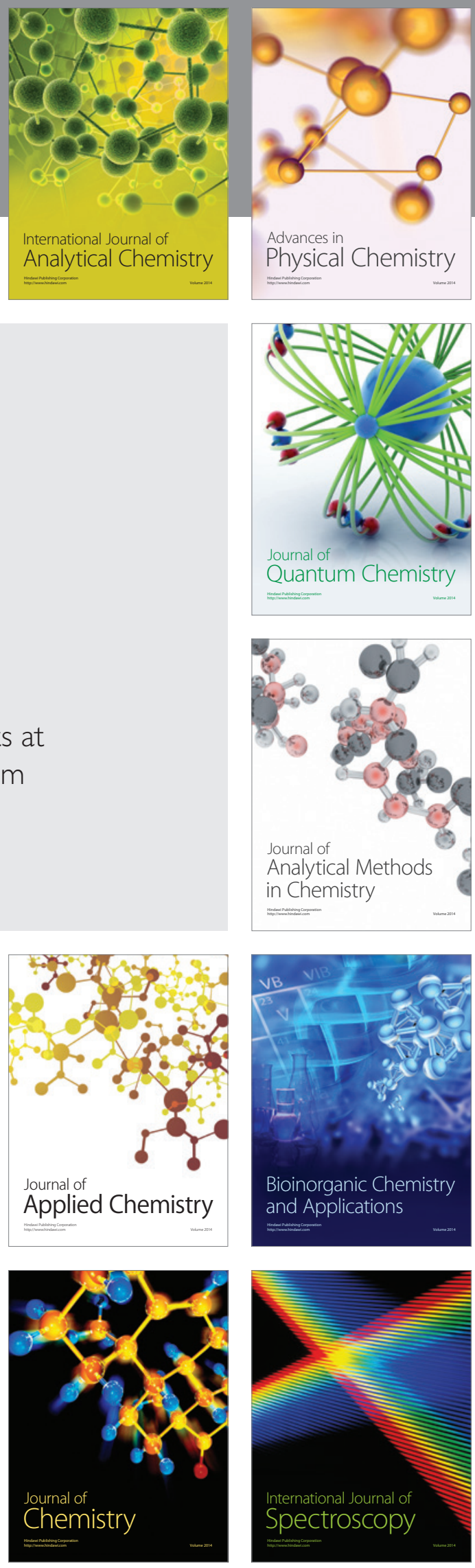